\title{
Towards Increasing the Compatibility of Student Pair Programmers
}

\author{
Neha Katira, Laurie Williams, Jason Osborne \\ Department of Computer Science, North Carolina State University, Raleigh, NC 27695 \\ \{nakatira, lawilli3, jaosborn\}@ncsu.edu
}

\begin{abstract}
As pair programming is used widely in software engineering education, instructors may wish to proactively form pairs to increase the likelihood of compatible pairs. A study involving 361 software engineering students was carried out at North Carolina State University to understand and predict pair compatibility. We have found that students are compatible with partners whom they perceive of similar skill, although instructors cannot proactively manage this perception. Pairing of two minority students is more likely and mixed gender pairs are less likely to be compatible. Additionally, pairing of students with similar actual skill level as measured by midterm grades in class, GPA, and SAT/GRE scores also likely results in compatible pairs. Our research addresses the following challenges faced by instructors in software engineering: 1) organizational concern in pairing of students; 2) increasing the retention rates of female and minority students in classes; and 3) proactively forming mutually-compatible pairs.
\end{abstract}

\section{Introduction}

Often we see that students are comfortable working in pairs. Occasionally, though, students are not compatible with their partners. This concerns the instructors. In our research, we were seeking patterns that predict pair compatibility. Our research addresses the following challenges faced by instructors in software engineering: 1) organizational concern in pairing students; 2) increasing the retention rates of female and minority students in classes; and 3) proactively forming mutually-compatible pairs.

We studied 361 undergraduate and graduate software engineering students at North Carolina State University (NCSU) during the fall 2002, spring 2003, and fall 2003 semesters. Our study was conducted in undergraduate Software Engineering (SE) and graduate object-oriented (OO) programming classes. Each of these classes had multiple two-to-three week assignments and the teaching assistants (TA) assigned pairing partners. Students never paired with the same partner more than once. Students evaluated their partners on a web-based peer evaluation survey ${ }^{1}$. The survey required the students to evaluate the contributions of their partner and the compatibility of the pair as perceived by the student. Our research focuses on the students' answers to the compatibility questions.

\footnotetext{
${ }^{1}$ Available for use by other instructors. The NCSU version can be found at http://arches.csc.ncsu.edu:8080/paireval/main
}

In Section 2, we outline the experiment and the hypotheses of our study. In Section, 3 we discuss the research motivation; in Section 4 we outline the results of our analyses, and finally we conclude in Section 5.

\section{Experiment and Hypotheses}

We studied students in SE and OO, as in Table 1. There were seven SE students who were both female and minority.

Table 1: Student distribution in the classes

\begin{tabular}{|c|c|c|c|}
\hline Class & No of Students & No. of Female & No Minority \\
\hline SE & 249 & 42 & 22 \\
\hline OO & 112 & 24 & N/A \\
\hline
\end{tabular}

This study builds upon our prior study [1] and focuses on finding correlations between the students' Myers Briggs personality type, actual skill, partner-perceived skill, a selfreported subjective measure of self-esteem, gender, and ethnicity demographics with the compatibility reported by students. Midterm grade, grade point average (GPA) and the SAT/GRE scores were the multiple measures of actual skill available to us. The under-representation of female and minority students [4] particularly interested us in analyzing their compatibility to help improve their experience in programming classes. Below we outline the hypotheses:

Pairs are more compatible if students with...

- $\quad .$. different personality types are grouped together.

- ... similar actual skill level are grouped together.

- ... similar perceived skill level are grouped together.

- ... similar self-esteem are grouped together.

- ... same gender are grouped together.

- ... similar ethnicity are grouped together.

SPSS $^{2}$ and $\mathrm{SAS}^{3}$ were used for the data analysis. Our most significant findings will be discussed in Section 4.

\section{Background and Research Motivation}

A survey-based study [3] performed at the University of Calgary and Southern Alberta Institute of Technology (SAIT) in Canada reported that students preferred working with a partner who matched their own qualifications and experiences. Results of a survey-based study at the University of Wales [6] revealed that students with lower self-esteem liked pair programming more than students with higher self-esteem. Students seemed to perform their best when paired with students of similar levels of self-esteem.

\footnotetext{
${ }^{2}$ http://www.spss.com

${ }^{3}$ http://www.sas.com
} 
A low representation of women and minority in computer science [4] intrigued us to analyze their compatibility. Female students generally dislike a myopic focus on the machine that detaches them from people [2]. A longitudinal study carried out at Carnegie Mellon University [2] revealed that male students are interested in "figuring out how it works", while female students attribute their interest in computer science to a larger societal framework.

Research shows that African-American success rates in science courses can be significantly improved by shifting the learning paradigm from individual study to one capitalizing on group processes [5]. Treisman's [7] "peer checking" experiment with African-American students at Berkeley reported that only about $4 \%$ of African-American students completing Treisman's collaborative "workshop" calculus class made a D or F, compared with $60 \%$ in earlier individual study versions of the course. A collaborative style of programming seems to appeal more to female and minority students because of the highlighted social nature of the pair programming paradigm. Factors influencing the compatibility of female and minority students will help in devising pairing schemes to improve their experience and possibly increase the likelihood of their retention in the field.

\section{Results}

In this section, we discuss our most significant results. Spearman's Rank Order (rho) correlation was used to analyze the correlation between pair compatibility and the factors of Myers Briggs personality type (MBTI), selfesteem, actual skill, and the partner perceived skill level. Table 2 shows $\mathrm{p}$ values of the correlation of compatibility with the factors. A p value less than 0.05 is considered statistically significant.

Table 2: Pair Compaibility Results

\begin{tabular}{|lccc|}
\hline & Female & Minority & All \\
\hline MBTI & 0.186 & 0.343 & 0.603 \\
Actual skill & $\mathbf{0 . 0 0 0}$ & $\mathbf{0 . 0 0 0}$ & $\mathbf{0 . 0 0 0}$ \\
Perceived skill & $\mathbf{0 . 0 0 0}$ & $\mathbf{0 . 0 0 0}$ & $\mathbf{0 . 0 0 0}$ \\
Self esteem & 0.131 & 0.325 & 0.393 \\
Gender & $\mathbf{0 . 0 0 2}$ & $\mathbf{0 . 0 0 9}$ & 0.051 \\
Ethnicity & $\mathbf{0 . 0 0 9}$ & 0.335 & $\mathbf{0 . 0 2 5}$ \\
\hline
\end{tabular}

These results indicate that students are compatible with partners whom they perceive of similar skill. Students in the software engineering classroom are likely to perceive compatibility with a partner who has a similar midterm grade and also a partner who has a similar SAT/GRE score. Pairing of female students with a partner who has a similar SAT/GRE/GPA will likely result in a compatible pair. Minority students perceive compatibility with a partner who has a similar GPA. Pairs with different gender are less likely to report compatibility. When both students in the pair are minority students, they are more likely to perceive compatibility. Minority students are comfortable working with students of the same gender. Generally, minority students are likely to perceive compatibility with their partner. These findings can be helpful to instructors in forming programming pairs.

\section{Summary and Future Work}

Through our research, we have found that the significant contributors in the compatibility of pair programmers are their perception of the partner's skill and also their actual skill level. Unfortunately, educators cannot be proactive about matching students based upon perceived skill. Mixed gender pairs are less likely to perceive compatibility. Students' personality type and self-esteem are not critical for their compatibility. Results from this research can help alleviate software engineering instructors' organizational concerns in pairing of students by increasing the likelihood of forming compatible pairs. Additionally, instructions can help improving the programming experience of female and minority students in hopes of increase their retention rates in the information technology field.

\section{Acknowledgements}

We thank Ms. Carol Miller, Ms. Suzanne Balik, Mr. Jason Schwarz, and Dr. Edward Gehringer - the instructors of the CS1 and the OO classes for their support in the collection of data. Special thanks to Xiao Ni from the Department of Statistics at NCSU for the helpful recommendations with SAS data analyses. We also thank the NCSU Software Engineering Reading Group for a careful review of the paper. This material is based upon the work supported by the National Science Foundation under the Grant No, 00305917. Any opinions, findings, and conclusions or recommendations expressed in this material are those of the author(s) and do not necessarily reflect the views of the National Science Foundation.

\section{References}

[1]Katira, N., Williams, L., Wiebe, E., Miller, C., Balik, S., Gehringer, E. On Understanding the Compatibility of Student Pair Programmers, Proceedings of ACM SIGCSE 2004, Norfolk, VI, pp. 7-11.

[2]Margolis, J., Fisher, A. and Miller, F., Caring about

Connections: Gender and Computing, IEEE Technology and Society Magazine/, Vol. 18 Issue 4, pp. 13 - 20, 1999

[3]Melnik, G. and Maurer, F., Perceptions of Agile Practices: A Student Survey, Extreme Programming/Agile Universe, Chicago, IL, 2002.

[4]National Center for Education Statistics: Digest of Education Statistics, 1990-2002, Institute of Education Sciences, U.S.

Department of Education, 1990-2002.

[5]Nelson, C., "Student Diversity Requires Different Approaches to College Teaching, Even in Math and Science," American Behavioral Scientist, vol. 40, pp. 165-175, 1996.

[6]Thomas, L., Ratcliffe, M. and A. Robertson, Code Warriors and Code-a-Phoebes: A study in attitude and pair programming, presented at SIGCSE, Reno, NV, 2003.

[7]Treisman, U. "Studying students studying calculus: A look at the lives of minority mathematics students in college.," The College Mathematics Journal, vol. 23, pp. 362-372, 1992. 